\title{
Nine
}

\section{THE FINAL ANTHROPIC PRINCIPLE}

According to another version of the Strong Anthropic Principle, the Final Anthropic Principle, our universe must exist. Why? Because it helps to create God. Our universe, together with infinitely many others, insures that God will one day come into being. This is its ultimate purpose and reason for being.

\section{The Omega Point as the Purpose of the Universe}

John Barrow and Frank Tipler develop and defend what they call the "Final Anthropic Principle," according to which the purpose of our presently Godless universe is to bring about God or the Omega Point. Only gradually do they identify the two, but this is the end result of their reflections, especially Tipler's. From the future, the Omega Point creates the world, but only after the world creates the Omega Point (if that makes any sense). God creates the world only after the world creates God.

According to Tipler, who identifies himself as an atheist, ${ }^{2}$ God's nonexistence is true now, but it will be false at the end of time when Theism becomes true. Tipler thinks that a godless universe will one day create God. The Anthropic Cosmological Principle culminates with the Omega Point. Tipler most fully develops the idea that the forthcoming Omega Point $=$ God in his The Physics of Immortality, 1994, where he contends that the Omega Point will raise us (or virtual cyberspace computerized emulations of us) from the dead billions of years from now and give our emulations eternal life. ${ }^{3}$

Every step in the futuristic pseudo-scientific eschatology of Barrow and Tipler is highly conjectural, unverified, and improbable. Over billions of future years, they believe, biological human life will perish; but it will re-embody itself in computerized robots that will gradually spread throughout the cosmos. Some day computerized humanity will exit planet earth and our solar system in space ships. Self-replicating humanoid or android computers will come to inhabit all of the Milky Way, then move on from there to conquer all other galaxies.

Barrow and Tipler remind us that eventually our solar system will cease to support biological life. Our sun has already burned half of its energy and has only five billion years to go. In four billion years, it will expand as a red giant to incinerate all its planets. Eventually, all stars/suns will exhaust their nuclear fuel, and all planets everywhere will become biologically uninhabitable in a cosmic heat death.

After biological human life as we know it is extinct, ${ }^{4}$ intelligent life itself will not end, Barrow and Tipler claim. They define life as "information processing,"s which, by definitional fiat, makes computers both alive and intelligent. (Yet, we must note, so much of the fullness of human reality and value is 
missing!) They insist that "Intelligent machines can be regarded as people. These machines may be our ultimate heirs." Eventually, we will construct complex robotic computers that can reproduce themselves and survive both the final cold of a closed universe's vast expansion and the final searing heat and pressure of its terminal contraction. In due time, all matter will decay, including protons and magnetic monopoles, so living computers will have to reembody themselves in radiant energy toward the end of the expansion phase. Toward the end of the contraction phase, they will be embodied in matter denser than iron and will endure long periods of hibernation with only fleeting moments of information-processing. Complex intelligent computers will still continue to communicate with one another and to gather information-until they take possession of, and make use of, all the mass/energy in the universe. Fat chance!

Similar life or information-collecting processes that include all possible histories are going on in infinitely many co-existing worlds in an all-inclusive Superspacetime. The Final Anthropic Principle agrees that the Principle of Plenitude as objectified in infinite worlds metaphysics caused our Big Bang. As our universe approaches its final singularity, it will merge with infinitely many other worlds that include all possible histories. Collectively, they will possess an infinite amount of information. The final Supersingularity in which infinite worlds merge in Superspacetime is the Omega Point. The whole meaning and purpose of our universe, of every universe, is to generate the Omega Point. Objectively, when the Omega Point is reached, "This is the end;"" but subjectively it will not be the end.

The Omega Point will be subjectively immortal because the pace of events will be slowed down so much that time will seem endless; (and presumably the then-Omniscient Omega will be too stupid to realize that it isn't). Barrow and Tipler tell us that "A modern-day theologian might wish to say that the totality of life at the Omega Point is omnipotent, omnipresent, and omniscient!"8 In The Anthropic Cosmological Principle, they do not actually say that the Omega Point is God; but since it exemplifies the traditional defining attributes of God, it is not misleading to say that according to the Final Anthropic Principle the purpose of the universe is to make God. Tipler explicitly calls the Omega Point "God" in later writings. ${ }^{9}$ Like Samuel Alexander, whose Space, Time, and Deity appeared in 1920, Barrow and Tipler predict that some day the universe will create God. Unlike Alexander, they also contend that God created the universe, since the future (God created by the universe) creates the past (God creating the universe). Anthropic Atheism results in Omega Point Theism in the very far distant future; Tipler's God "exists mainly at the end of time."

\section{Critique of the Final Anthropic Principle}

The incredibly conjectural predictions proffered by Barrow and Tipler make their Final Anthropic Principle (FAP) highly problematic. Not inappropriately, 
one critic, Marvin Gardner, renamed their principle the "Completely Ridiculous Anthropic Principle (CRAP)"."

\section{A. Unfounded Assumptions}

Not one of the following claims made or presupposed by Barrow and Tipler are known to be true, and most of them are either meaningless, blatantly false, immensely improbable, or logically incoherent.

1. Human beings (or our robot computer descendants) will one day travel to all inhabitable planets in the Milky way and eventually to every galaxy in the universe. This presupposes that:

a. Human minds and bodies can either survive the weightlessness and other adversities of very long-distance space-travel; or they can be perfectly encoded in lightweight computers that can survive such rigors. future.

b. Human beings will invest heavily in space-research and travel in the

c. Cheap and abundant sources of energy for space travel will be available in the future.

d. We and our biological descendants will long survive the enormous genocidal propensities of our species, our unpredictable adventures and misadventures with nuclear energy, our incredibly short-sighted environmental destructiveness, our dabbling with bioterrorism, and our propensity to overpopulate the earth.

e. Many planeis throughout the Milky Way and the rest of the cosmos are inhabitable and will provide suitable habitats for our computerized robot descendants.

f. If inhabited, the occupants of other planets will be receptive to computerized humanoid aliens.

g. The native bacterial, viral, chemical, physical, and social occupants of other inhabited planets will not be devastatingly hostile to and destructive of computerized humanoid aliens.

The list could go on and on.

2. Life is nothing but "information processing," which implies that automobiles and all other machines are alive. ${ }^{12}$

3. Machines (information processing computers) are people; they are (or will be) just as conscious, intelligent, and valuable intrinsically as biochemical, carbon-based human beings.

4. We and our biological descendants can be persuaded that 2 . is true, will come to care about the long-term destiny of android computers, and will recognize that the whole meaning of our existence depends on what happens to merged computerized robots billions of years from now.

5. Our universe, and each member of the oscillating set to which it belongs, contains enough mass/energy to close it, so its expansion phase will halt, 
and the terminal Big Crunch will coalesce into the Omega Point. This is also true of infinitely many co-existing worlds and/or oscillating sets of worlds in Superspacetime. The Omega Point will result from the merger of an infinite number of universes that actualize all possibilities.

6. Intelligent computers can survive and function in the extremely hostile physical conditions that will prevail toward the end of the universe's expansion and contraction phases. At the end of time, they and the information they encode can survive the infinite density, timelessness, and spacelessness of the ultimate singularity, the Omega Point.

7. Computers can and will eventually capture and make use of all the mass/energy in the universe and manipulate its evolution.

8. Processes like 1-7 above are going on in infinitely many worlds coexisting with ours in infinite Superspacetime. Infinitely many worlds actualize all possibilities.

9. Near the final state of the Superuniverse, infinitely many computercaptured universes will merge with ours to form the Omega Point. Their contraction phases will somehow coincide with that of our world or its ultimate oscillating successor. Infinitely many universes can and will find ways to contract and merge that preserve and are not destructive of information about every detail of their existence.

10. The duration of the Omega Point will be objectively finite, but it will be subjectively immortal-or too slow and stupid to know that it is mortal!

11. At the end of all spacetime, the Omega Point, God, will become omnipotent, omnipresent, and omniscient.

The possible and the probable are not identical, and the logically incoherent is not even possible. None of the above claims are known to be true, and most if not all of them are highly improbable, blatantly false, unintelligible, or otherwise implausible. No attempt will be made here to criticize these claims in depth, but many of these presumptions have been decisively refuted in earlier discussions. The absurdity of this position speaks for itself.

Let us consider only two of many insuperable obstacles to the realization of Star Trek, Star Wars, and all fanciful futuristic human space-travel scenarios-the time involved in space-travel, and the incredible quantities of energy required. As Timothy Ferris indicates,

The stars are just too far away: A spacecraft capable of traveling a million miles per hour-and this would be a stunningly fast ship, one that could fly from Earth to Mars in less than an hour-would take nearly three thousand years to reach Alpha Centauri, the nearest star."13

Alpha Centauri is 4.3 light years from us; it actually consists of three stars closely encircling one another; it probably boasts no habitable planets, for any that try to form would be ground to bits by this encirclement. 
In his 1994 book, Tipler concedes all of this, then opts for travel beyond Alpha Centauri to two stars resembling our sun that are 11.3 and 10.7 light years away as the first feasible extra-solar destination. Habitable asteroids might exist along the way, he hopes. Tiny microcomputers could be transported to these stars at 90 percent of the speed of light in only twelve years or so, he claims. ${ }^{14}$ But how and when will we build spaceships that can travel at 90 percent of the speed of light? Surely not in twelve years. Probably never.

Building space ships to carry tiny microcomputers is one thing; building them to carry human beings is another. This is where the available energy problem gets serious. Physicist John A. Jungerman tries to bring Trekkies back to sober realism about this. Flying the starship Enterprise to the nearest star at only half the speed of light (much faster than in Ferris's example above) would take eight years one way, but accelerating it to that speed would be absolutely prohibitive. At four million tons of mass, the energy (fuel) required to accelerate the Enterprise to half the speed of light for the trip would be

the energy equivalent of about $10^{16}$ tons of TNT, or ten thousand trillion tons! The nuclear arsenals of all countries contain about twenty billion tons of TNT equivalent. So to put this into perspective, the fuel required for the acceleration to half the speed of light would be the energy equivalent of about five hundred thousand times all the nuclear arsenals of the world. ${ }^{15}$

Jungerman further indicates that when the Enterprise gets to the nearest star, an equal amount of energy will be required to slow the ship down and land it. And this says nothing of fuel required for the return trip! Fictional space warps that allow for overcoming these obvious limitations of space-travel remain "a science fiction dream," Jungerman cautions. ${ }^{16}$

Ferris, Tipler, and Jungerman discuss only space-travel within our galaxy. Intergalactic travel, the real stuff of science fiction, is conspicuously less feasible. The light we see from the "nearby" Andromeda Galaxy has been traveling (at the speed of light) for over two million years to reach us. If you plan to vacation some day in the Andromeda Galaxy, forget it! Even if you would not age much while traveling at almost the speed of light, assuming you could attain that speed, you would still take over two million earth years to get there. We cannot conceive of the obstacles that are likely to arise or the fuel required for such a trip. The only intelligent space travelers likely to migrate to other solar systems or galaxies will be human-made computers; they won't be us or our biological descendants. Even if a chosen few elite specimens of biological humankind eventually travel to and survive in extraterrestrial environments, most ordinary people (the likes of you and me) will be left behind to suffer the fate of this fragile earth, whatever that fate may be. If we don't make it here, we won't make it anywhere! 
Some futurists paint a much rosier picture of our prospects for space travel. Whether it will ever happen or not is really quite irrelevant to the central issue, which is: Upon what does living a meaningful life depend? Are our lives meaningless if the ultimate destiny of our species is tied to our fragile planet earth, or to our solar system? Are our lives meaningless if neither we nor our biological descendants will ever colonize other planets, first within and then beyond our solar system, and then beyond our galaxy? Is every person's life meaningless unless he or she makes some contribution to space travel and the "terraformation" and colonization of other planets? Some astrophysicists think so, as indicated next.

Suppose, as seems likely, that neither we nor our biological descendants will ever get beyond our solar system, or that even if we do only a tiny number of human beings will ever colonize other planets. Does this mean that most of us live meaningless lives? Suppose that the earth ultimately dies a "heat death" and that this is true of all the other planets that a few elite human beings might ever inhabit. Will it all be for nothing? The answer is "yes" if the worth of our lives depends on endlessly perpetrating our species or contributing to some ultimate transspecies objective. The answer is "No" if our lives have intrinsic meaning and worth here and now.

\section{B. The Meaning and Value of Human Life}

Philosophers identify at least two types of value or goodness-intrinsic, and instrumental or extrinsic. An intrinsic good is an end in itself, valuable for its own sake. An extrinsic good is an efficient means to some other goal or value beyond itself. Extrinsic goods have desirable consequences. Systemic goodness-the value of concepts, ideas, and formalities of every description, was recently added to this traditional duality of goodness by Robert S. Hartman. ${ }^{17}$ In considering the value of individual human lives, we must decide whether we have intrinsic worth, extrinsic worth, systemic worth, or some combination of all three.

The word "meaning" may have many different meanings, but it is something conceptual, something systemically good, as is "a meaningful life." Meaningful lives and valuable lives are intimately related. All valuable lives are meaningful, but they are not merely conceptual. Let us specify that human life is meaningful if we can conceptually comprehend and wholeheartedly affirm its intrinsic, extrinsic, and systemic worth and can understand how human values are supported by broad social, physical, psychological, cosmological, and metaphysical structures and environments. Support makes little sense unless threats and dangers exist, so these too must be factored into any conceptual scheme that captures the meaning of human life. Metaphysical support for human life will be covered in following chapters. Valuable individual human lives are the concrete realities to which our concepts of "meaningful lives" make reference. 


\section{i. Human Existence as Merely a Means to Something Beyond}

Advocates of the Final Anthropic Principle make some commonplace but highly questionable assumptions about the value and meaning of human life; and they incorporate their flawed assumptions into their interpretation of the FAP. Barrow and Tipler suppose that the meaning and value of human life (of all kinds of life, for that matter) lie entirely in its future consequences. This implies that human lives here and now are nothing more than instrumental goods, that our own lives have no inherent or intrinsic meaning and worth at all. The following excerpt expresses this key axiological assumption presupposed by the Final Anthropic Principle. According to Barrow and Tipler,

We know space travel is possible. We argued that even interstellar travel is possible. Thus once space travel begins, there are, in principle, no further physical barriers to prevent Homo sapiens (or our descendants) from eventually expanding to colonize a substantial portion, if not all, of the visible Cosmos. Once this has occurred, it becomes quite reasonable to speculate that the operations of these intelligent beings could begin to affect the large scale evolution of the Universe. If this is true, it would be in this era-in the far future Near the Final State of the Universe-that the true significance of life and intelligence would manifest itself. Present-day life would then have cosmic significance because of what future life may someday accomplish. ${ }^{18}$

If the "true significance" of life and intelligence is manifested only in the very distant future, this means that it has no true significance here and now. If present-day lives have significance only then or as means to then, they have no significance now, except as extrinsic goods. The end to which we are mere means lies billions of years in the future, the Omega Point. But the promise of the Final Anthropic Principle is as hollow as the sign in the bar that says "Free Beer Tomorrow," for tomorrow never comes. Biological human life will be totally extinct for billions if not trillions of years before it has any "true significance."

Tipler makes the purely extrinsic or instrumental worth of human life even clearer in a later essay where he asserts that (1) "Value is something connected with life, and thus if value is to remain in the universe, life must persist indefinitely." "(2) A universe "in which life (and hence intelligence) and all its works disappeared forever would in my judgment be ultimately meaningless. ${ }^{20}$ (3) The laws of physics ultimately doom the human species to extinction. ${ }^{21}$ (4) "Humankind's place in the scheme of things is that of an intermediate link."22 (5) The future of life belongs to computers, not to DNA (biological) based life. ${ }^{23}$ In his 1994 book, Tipler adds that the intelligent computers of the future will eventually raise us-or rather, computerized virtual cyberspace emulations of 
us-from the dead; and we will be identical with these virtual emulations. ${ }^{24}$ Do you see any problems about personal identity here? Does the Final Anthropic Principle make you feel exploited as a mere means to ends beyond yourself, ends that you do not embrace?

\section{ii. Enduring Grand Objectives}

Many science-minded cosmologists feel and express deep anxiety about the meaning and value of human life because, like Barrow and Tipler, they presume that our lives are utterly worthless unless they contribute significantly to the achievement of some Enduring Grand Objective located in the far, far distant future. Unlike Barrow and Tipler, many skeptical or pessimistic cosmologists are convinced that no Enduring Grand Objective like the Omega Point will ever exist. So they despair.

Cosmological pessimists fully comprehend that humankind is destined to perish some day in a hostile cosmic environment, and they are obsessed by an awareness that our lives are extraordinarily tiny and brief within the vastness of cosmic spacetime. They conclude that human life has no value or meaning, and neither does the universe. This sort of cosmological pessimism was well expressed in an often-quoted excerpt from Steven Weinberg's book, The First Three Minutes. According to Weinberg, "Whichever cosmological model proves correct, there is not much comfort in any of this." Reflecting on the beautiful and supportive earthly environment in which we live, Weinberg comments,

It is very hard to realize that all this is just a tiny part of an overwhelmingly hostile universe. It is even harder to realize that this present universe has evolved from an unspeakably unfamiliar early condition, and faces a future extinction of endless cold or intolerable heat. The more the universe seems comprehensible, the more it also seems pointless. ${ }^{25}$

All twenty seven contemporary cosmologists interviewed by Alan Lightman and Roberta Brawer for their Origins: The Lives and Worlds of Modern Cosmologists were asked to comment on this quotation from Weinberg. ${ }^{26}$ Their responses are fascinating! Clearly, many contemporary cosmologists share Weinberg's cosmic pessimism, and he did not change his mind in his more recent Dreams of a Final Theory, 1992. ${ }^{27}$

\section{iii. Human Insignificance in the Grand Scheme of Things}

What should we make of all of this? The universe and our own existence are likely to seem pointless if we make any or all of the following assumptions:

1. No Enduring Grand Objective will ever exist. 
2. Genuine value and meaning correlate with enormous physical size and immense temporal duration or permanence. Little things have little worth!

3. Compared to the universe, individual human lives are indeed small and brief, and all human (and nonhuman) life in this world will eventually be extinguished.

Although 3. is true, 2. is not; and 1. is irrelevant to the question of the meaning and value of human existence.

Biological human life is indeed spatially tiny and temporally short when compared with the vastness of the spatiotemporal cosmos. Like Weinberg, Stephen Hawking also contrasted the immensity of the universe with "insignificant creatures like ourselves." ${ }^{28}$ In the 1990 BBC television program Master of the Universe, Hawking said that

We are such insignificant creatures on a minor planet of a very average star in the outer suburbs of one of a hundred thousand million galaxies. So it is difficult to believe in a God that should care about us or even notice our existence. ${ }^{29}$

Many individuals who reflect on our position in the universe seem to equate worth with size. Even the theologian John Hick proclaims that "a naturalistic conclusion" is strongly supported by the "sheer size" of the universe and "humanity's correlatively minute place within its spatial and temporal immensity." 30 Victor J. Stenger acquiesces: "So small is humankind" and "So vast is the universe," he laments "The insignificance of humanity is almost impossible for most humans to accept." "Surely the universe does not care about human existence," ${ }^{\prime 32}$ Stenger bemoans. Frank Tipler, who locates ultimate meaning in the Omega Point, argues that we humans live "at an exceedingly early time" in the history of the universe, that "Most of life is in the future," and that "It is our relative insignificance in time, not space, which is the real challenge posed by modern cosmology for traditional religion." 33 Temporalistic versions of the insignificance argument focus on how little of the cosmic time line is occupied by living things. ${ }^{34}$

Cosmologists with this pessimistic mind-set often assume that attributing great worth to individual human beings is merely a human judgment, therefore untrustworthy; but note carefully that the judgment that we do not have great worth is also merely a human judgment! The real problem is that the connection these pessimists assume between size, duration, and significance is all very wrong, both cosmologically (spatiotemporally) and axiologically (valuationally). Cosmologically, Anthropic Cosmologists demonstrate, human life could never evolve in a universe very much smaller or younger than our own. Temporally, producing complex life requires billions of years for generations of supernovae to come and go and billions more for complex forms of life to evolve locally. Spatially, a stable and hospitable solar/planetary system requires vast separa- 
tions between heavenly bodies if life-budding, life-building planets are to avoid being torn out of their orbits by the gravitational effects of passing stars or other planets. ${ }^{35}$ Spatial versions of the insignificance argument center on how tiny our bodies are in relation to the vastness of space, temporal versions on how briefly we endure within the whole of time.

Axiologically, our species and our individual lives are not insignificant simply because of our relatively limited physical size and duration. Value and meaning in and for our lives do not correlate with or depend upon great magnitude and/or permanence. Our species has existed for only a fraction of the age of the universe. As adults we typically live only seventy years or so, weigh between one and two hundred pounds, are only about six feet tall, and are less than two feet wide. There are exceptions! By comparison with the totality of time and space, we are tiny and trivial indeed; but that is the wrong comparison, a childish comparison, uninformed by the study of value theory. Philosophers tend to be scientifically naive; but scientists doing cosmology tend to be philosophically naive!

\section{iv. The Intrinsic Worth of Human Existence}

Philosophers deeply ponder the question of whether anything has intrinsic worth, is valuable in and of itself; and after careful consideration they usually find immense intrinsic worth in human existence. Few philosophically astute value theorists would agree with pessimistic astrophysicists that our speciestypical spatiotemporal limits are incompatible with immense intrinsic worth, for they reject the premise that value depends on immense size and duration. We don't have little worth just because we occupy very little spacetime!

If and when we are not blinded by cosmological magnitude, we can readily appreciate the great intrinsic worth of even small human infants, to say nothing of larger adults. When loving an infant, we realize that within broad limits smallness is no obstacle to great significance. Teilhard de Chardin was right (in part) in affirming that significance depends on the complexity of consciousness. ${ }^{36}$ It also depends on the uniqueness of that consciousness, and on properties experienced and actualized by and within that consciousness. Philosophers who reflect deeply on the question usually conclude that nothing has much intrinsic worth, if any, apart from consciousness; but more than mere, pure, or complex consciousness as such is required for life to have meaning and value.

Physically we are tiny, and our lives are short compared to the cosmos as a whole. Yet, we complex, conscious, and unique human beings are immensely if not infinitely valuable or significant in and for ourselves. Our great inherent worth does not depend upon our size, our duration, or upon what we produce in the near or distant future. This is true also of all but the simplest animals, but that is another story, too long to tell at present. We are immensely complex conscious individuated creatures, despite our spatiotemporal limitations. We can 
even comprehend the Big Bang and our place within the universe! Human consciousness and the physiological conditions that support it are incomprehensibly complex. Nothing else in the world known to us matches the intricacy of the human brain. Intrinsic value depends heavily on the enormous complexity and activity of embodied consciousness; and all but the most unfortunate human individuals have it or eventually achieve it. Great intrinsic worth depends on complexity of individuated consciousness; it also requires just the right kinds of intricacy, activity, and contents. But intrinsic value is quite independent of vast physical size and endurance. Some size and endurance are essential for spatiotemporally embodied individuated consciousness, but not vastness.

Intrinsic value is complex. Complex individuated consciousness as well as just the right activities and intensional objects of consciousness are required for great intrinsic worth. Intrinsic human goodness or worth is a synthesis of unrepeatable, unique, individuated consciousness with many additional repeatable concretized universals that are dynamic, conceptual, emotional, affective, and volitional. Many philosophers and other thoughtful persons realize that brain-grounded individuated consciousness can be enriched positively in many repeatable ways. Positive consciousness-enrichers or enhancers include the pursuit and attainment of happiness, knowledge, moral and religious virtues, love, interpersonal intimacy, beauty in art and nature, adventure, creativity in every constructive domain of human interest, and fulfillment of our beneficial or positive capacities, needs, desires, interests, and purposes.

Our worth as conscious individuals does not consist merely in our being instrumental receptacles for the realization of these universal and repeatable abstractions, as many philosophers traditionally assumed. Individuated consciousness is not just a worthless or merely useful bucket into which intrinsically valuable concrete universals may be poured. Individuated consciousness has its own inherent worth; and our fullest intrinsic worth consists of a synthesis of our individuated consciousness with the consciousness-enriching universals just mentioned.

In the existing cosmos, with a bit of luck and many wise choices, consciousness-enriching activities and goods can be and are available to individual human beings in great abundance, despite our spatiotemporal finitude. Embellished by such repeatable good-making properties, complex individual embodied consciousness is immensely and inherently good. It is immensely significant, meaningful, and valuable in and of itself right here and now, even with all its obvious faults and limitations. ${ }^{37}$ The universe has a magnificent point simply because we are here, no matter what comes later. For each of us, our being here is a valuable end in itself. Our worth does not depend entirely on our being means to ends beyond ourselves, grand or otherwise.

Enduring Grand Objectives are hypothetical goods that arrive later and last for eons of time if not forever. If our universe and our individual lives have no Enduring Grand Objective, can we or our universe be anything but pointless, 
given the inevitable eventual extinction of our individual lives and of humankind itself? According to pessimists like Weinberg and Hawking, no Enduring Grand Objective will ever arrive, and they despair.

Barrow and Tipler, by contrast, situate the whole point of human existence in their own peculiar Enduring Grand Objective, the Omega Point. Omega will arrive (supposedly) at the end of our (supposedly) closed universe; and it will live happily and omnisciently ever after (subjectively).

Barrow and Tipler are not alone in locating the meaning of human life in some enduring Grand Objective; but grandiose objectives may be conceived in many different ways. Freeman J. Dyson finds our existence to be ultimately meaningful only if and because the existence of intelligent life can be prolonged infinitely in an open universe through radical biological adaptations; ${ }^{38}$ but this just turns the infinite prolongation of intelligent biological life into another Enduring Grand Objective, another free beer tomorrow that never comes.

For traditional Christianity, the endless survival of individuals after death in Heaven or the Kingdom of God, however conceived, is the Enduring Grand Objective. Most Christians, following St. Paul, assume that life here and now is completely meaningless, an utterly pointless pilgrimage to nowhere, unless there is a resurrection and a Heaven. Heavenly survival after death is the whole point of human existence, without which everything is in vain. Traditional Christianity assumed and taught that life in this world has value and meaning only as a necessary condition for or means to a Grand Existence Beyond this World.

Suppose, however, that no Omega Point will ever arrive; our descendants will never space travel to far distant solar systems and galaxies; the existence of intelligent biological life will not be infinitely prolonged; and we as individuals will not survive after death. Would human life really be worthless, pointless?

All Enduring Grand Objective theories mistakenly locate the meaning and value of human life only or primarily in some distant future Grand Objective that will last indefinitely; but what gives these Enduring Grand Objectives their significance? Nothing more than exactly the same conditions-perhaps intensified, amplified, and prolonged-that make active, individuated, and enriched consciousness so precious here and now! Grand Objectives themselves have value and meaning only to the extent that they epitomize things that are presently valuable and meaningful. If life now is a purely instrumental pilgrimage, life in some glorious future can be only an instrumental means to some even Grander Objective that never comes. If no inherent meaning and goodness now exist, no enduring and glorified future version of it will have any.

Future meaning and goodness make no sense unless meaning and goodness are now available. Even without a "then," intrinsic worth exists now. Individuated conscious life is for living-for itself; and its intrinsic worth lies in living it. The meaning and value of our existence do not depend on some future Enduring Grand Objective, even if there is one. The significance of either 
Heaven or the Final Anthropic Principle is greatly exaggerated, even if something like it is true.

What makes the Omega Point so valuable according to Barrow and Tipler? Things like power, presence, and knowledge (omnipotence, omnipresence, and omniscience)! But we have these and many other excellences now, not in their "omni" form, but sufficiently to make conscious life in this world a great gift, an immense if not an infinite good in, of, to, and for itself. Why are other Enduring Grand Objectives like immensely prolonged intelligent, affective, volitional consciousness in this world or in the next valuable? Isn't it because they contain the very same good-making properties that make our lives here and now so precious-active individuated consciousness enriched by happiness, knowledge, adventure, and their pursuit, by moral and religious virtues of many descriptions, by love, friendship, and other manifestations of interpersonal intimacy, by beauty in nature, society, and the arts, by creativity in every constructive domain of human interest, and by fulfillment of our beneficial capacities, needs, desire, and purposes? Value and significance-making properties and predicates may not be available in the same degree here and now as they would be in those idealized Enduring Grand Objectives in which we continue to survive after death, but they can be and usually are quite sufficient to warrant cherishing our present lives for their own sakes-not just as a means, a pilgrimage, to something beyond themselves.

\section{v. The Meaning and Value of Infinitely Prolonged Existence}

Hell would be infinitely prolonged individuated consciousness devoid of all value-enhancing enrichments including hope, together with the everlasting presence of their bad-making opposites. Its meaninglessness would consist largely in knowing that good-making properties are not and will never be supported by Hell's broad environment. Infinite duration (immortality or resurrection) and infinite complexity or richness in properties as such fail to differentiate Heaven and Hell beyond this life from heaven and hell on earth. Quality of existence matters immensely here and hereafter, not just quantity.

Both quality and quantity (intensity and duration) of consciousnessenhancing properties are important; but individuated consciousness can be "enriched" numerically with bad-making properties like misery, authoritarian animosity to knowledge, cowardly adventure-avoidance, innumerable moral and religious vices like hatred, resentment, selfishness, interpersonal insensitivity, philistine revulsion to beauty and the arts, and fulfillment of welfare-destructive interests, desires, and purposes. Quantitative "enrichment" of complex individual consciousness with bad-making properties results in intrinsic disvalue, not intrinsic goodness. Since bad-making properties may in principle be indefinitely prolonged, neither property-richness nor temporal endurance suffice for intrinsic goodness and meaningful existence. Intrinsically good lives involve both 
qualitative and quantitative enrichment of conscious experiences and activities with good-making properties, not bad-making properties; and meaningful lives involve conceptually understanding that the intrinsic, extrinsic, and systemic goodness of our lives is supported by our broad environments. Kind as well as number and complexity of properties enter into the constitution of inherent worth and meaningful existence, but vast spatiality or temporality are irrelevant, as are far distant Grand Objectives.

Sheer endurance is really not as valuable as supposed by those who compare our brief lives with the duration of the universe, or who yearn for everlasting survival after death. Sheer immortality or infinite endurance should not be confused with infinite goodness and meaning. According to traditional western religion, immortality-infinite endurance-could co-exist with and consist of nothing but endless evil and senselessness. Hell is forever. Nothing could be worse or more meaningless than everlasting survival, infinite duration, immortality, in a traditional Christian Hell.

Hell could be infinitely prolonged and infinitely complex or rich in properties, but Hell nonetheless! How so? As John Stuart Mill maintained, many different qualities of feeling are called "pleasure," and many others are called "pain." The agreeable feelings we derive from reading our favorite authors are qualitatively different from the pleasures of music, dining, sex, or sadism. Likewise, the disagreeable feelings that we get from reading Schopenhauer and Nietzsche (I wanted to say Kant!) are quite distinct from the pains of grief, guilt, loneliness, boredom, emphysema, a bee sting, and/or a severe injury or burn. ${ }^{39}$

Consider the relation between pains and endless duration. Mill gave little attention to the topic of qualitatively distinct pains, and he wasted no energy worrying about Hell. But, for the fun of it, let us ask how many different kinds or qualities of pain might exist and consider how a concept of Hell might be constructed from such information. The issues are partly empirical and partly logical. Logically, an infinite number of qualitatively distinct kinds of pain might exist. Hell, says traditional Christianity, is a place of infinite pain in multiple respects. First, Hell could be infinitely rich in distinct qualities of pain; next, each of these could be infinitely intense; finally, all of these combined could endure forever, unrelieved. Working out all the details would give us a modern version of Dante's Divine Comedy and John Milton's Paradise Lost!

So conceived, would the existence of conscious persons enduring infinitely complex and intense pain endlessly in Hell have any positive meaning or intrinsic worth? Surely not! Total extinction would be preferable by far, and part of Hell's misery supposedly lies in the realization, the conceptualization, that extinction is impossible. Hell would be so devoid of hope that it allows for no hope for extinction! A brief moment in such a Hell would be unthinkably horrible, and an infinite duration of it would be infinitely bad, endlessly meaningless. Obviously, immense or endless endurance (immortality) does not necessarily correlate with positive worth and meaning! 
Both Heaven and Hell are at least logically possible as parallel or coexisting universes, but whether or not they actually exist we are not likely to know for sure in this life-unless we are confident that all possible universes are actualized. Still, nothing could be better, more meaningful, than infinite duration or immortality in a traditional Heaven, where individuated conscious existence is supposedly as rich in good-making properties as it can bear-within a totally supportive environment. Perhaps individuated consciousness and its excellencies do not endure forever; but Heaven might be nice anyway, since more of a good thing is generally better than less. To those who say that we would become too bored to want to exist after a vast period of heavenly survival, the proper reply is that we would be happy to try it for a few million years just to find out! Love, kindness, joy, creativity, curiosity, learning, and growth seem to be inexhaustible forever.

To return to the here and now, positive enrichments that make presently existing individuated consciousness so enormously valuable to, for, and in itself also make it instrumentally valuable to other persons (and to God, as explained in the next chapter). Our intrinsic and systemic worth here and now, and our extrinsic helpfulness and usefulness to others, are precious gifts that we contribute ultimately to God, who remembers and cherishes our intrinsic, extrinsic, and systemic worth forever. Others can recognize our intrinsic worth, as we can theirs, and relate respectfully to us as mutual members of a kingdom of ends, a kingdom of God. Our worth to others consists in what we mean to them here and now and the impact that we have on the quality and duration of their lives; but it has nothing to do with spatiotemporal vastness, or with an ultimate Omega Point, or with the interminable survival of intelligent biological or cybernetic beings, or with any other Enduring Grand Objective.

Conscious human existence here and now can become intrinsically disvaluable, something to be avoided or eliminated for its own nasty sake. For example, all of a terminally ill person's wakeful moments may be filled with overwhelming and unrelievable suffering, despite medicine's best efforts to provide pain relief, and he or she may beg for a merciful death. Along with many others, I believe that moral duty requires the expeditious and active elimination of such intolerable and immense intrinsic disvalue, especially when death is requested by hopelessly ill persons crushed by unrelievable bodily pain and/or mental distress. With good pain management, fortunately, most suffering is relievable today; but not all. Active voluntary mercy killing is sometimes a moral duty, although not yet legal in most countries. Intrinsic disvalue for individuated consciousness is not mere privation or deficiency in kind or number of properties. It involves the presence of properties that are undesirable and worth avoiding or eliminating for their own nasty sakes-like the excruciating and unrelievable sufferings that maliciously tortured victims, or terminally ill patients, themselves judge to be too horrible to endure. 
If something is intrinsically good, its value does not depend on its consequences, even if they, too, are desirable. Life for us here and now, properly enriched, is an immense intrinsic good; and its inherent meaning and goodness are completely independent of any and all Enduring Grand Objectives or spatiotemporal amplitudes. With or without resettlement of biological or computerized human beings on other planets, or a traditional Heaven, or an Anthropic Omega Point, human and animal life can, and usually do, have immense and intrinsic significance and worth in themselves here and now. The goodness or worth of enriched, conscious, individual existence is inherent, in itself, and not merely instrumental. As Ralph Waldo Emerson said, "My life is for itself and not for a spectacle. ${ }^{" 40}$

More of a good thing is generally better than less; but more goodness requires more time-for ourselves and/or for posterity. It would be nice to know that our descendants will happily inhabit this good and beautiful earth, rich in life-forms, for many generations. We really are capable of caring deeply about future generations and for non-human species; but do we actually care enough? We tend to be very short-sighted and to consume wantonly, wastefully, and conspicuously the natural resources that future generations, human and nonhuman, will need for worthwhile lives; and we overpopulate the earth with other people who bear the same imperfections. We pollute our supportive environment so much with chemical and nuclear poisons that the earth may be uninhabitable in a few generations by almost everything except cockroaches. Stephen Hawking now believes that the greenhouse effect resulting from human endeavors that spew excessive carbon dioxide into the atmosphere will make the earth uninhabitable in less than a thousand years; and he pushes space travel and colonizing other planets so that a few of us will survive. ${ }^{41}$ The trouble is, only a very few can survive this way; most human beings, most of our descendants, will perish with the dying earth. Does a species that befouls its own nest while knowing better really deserve to survive? Does our own species deserve to survive, given our enormous and largely unrestrained propensity to make the earth uninhabitable for our own kind and for all other forms of life? If we are stupid, greedy, and shortsighted enough to contaminate this fabulously beautiful planet earth to the point of uninhabitability, Homo sapiens does not deserve to survive. We must yet prove that we do.

To summarize, the Final Anthropic Principle affirms that infinitely many worlds, including our own, exist for the purpose of bringing about the Omega Point or God. Life and intelligence will gradually spread throughout our cosmos and all others. Biological life will be replaced eventually by computerized, robotized, android intelligence. In the far distant future, all universes, ours included, will merge into the Omega Point, an Ultimate Supercomputer that at the end becomes omnipotent, omnipresent, omniscient, and Divine. The Omega Point includes the full actuality of all possible histories, all possible universes, rolled up into one. Barrow and Tipler make many dubious assumptions concern- 
ing the survival and proliferation of intelligence. Most seriously, they are mistaken about the meaning and value of the lives we now enjoy. They presume that our lives have no immediate intrinsic meaning or worth, and that our true significance will arrive only with the Omega Point-trillions of years after our extinction.

Enduring Grand Objectives are attractive only because they amplify and prolong all the good things that make life here and now meaningful and worthwhile. Because our lives here and now are ends in themselves and not mere means to ends beyond themselves, their true significance is here and now; and the vastness of the universe is irrelevant. The value of life is in living and enriching it-intrinsically, extrinsically, and systemically. The meaning or worth of our existence depends in no way on some remote Enduring Grand Objective, even if one will eventually come to be; but meaning is, or would be, enhanced significantly if and when we know that our worth is supported by our ultimate metaphysical environment, God, in ways explained in the following chapters. 